\title{
THE NONOCCURRENCE OF THE LAVRENTIEV PHENOMENON FOR A CLASS OF VARIATIONAL FUNCTIONALS*
}

\author{
GIOVANNI BONFANTI ${ }^{\dagger}$ AND ARRIGO CELLINA ${ }^{\dagger}$
}

\begin{abstract}
We prove that the Lavrentiev phenomenon does not occur for the functional $\int_{\Omega}[l(\mid \nabla$ $u(x) \mid)+G(u)] d x$ on $u-u^{0} \in W_{0}^{1,1}(\Omega)$, where $L$ and $G$ are convex functions subject to additional regularity assumptions, and where $\partial \Omega$ and $u^{0}$ are smooth.
\end{abstract}

Key words. calculus of variations, Lavrentiev phenomenon, boundedness of solutions

AMS subject classification. $49 \mathrm{~K} 10$

DOI. $10.1137 / 12086618 \mathrm{X}$

1. Introduction. In 1927, in a paper by Lavrentiev [6], an example was shown of a phenomenon, later called the Lavrentiev phenomenon, where the infimum, over the set of absolutely continuous functions, of a functional of the calculus of variations was strictly lower than the infimum of the same functional over the set of Lipschitzean functions satisfying the same boundary conditions; since that time, finding conditions that would imply the nonoccurrence of the phenomenon has been of some interest. Alberti and Serra Cassano in [1] proved that, when the integration set is one-dimensional, the phenomenon does not occur for autonomous Lagrangians. For functionals on a higher dimensional integration set $\Omega$, conditions for the nonoccurrence of the phenomenon were considered in [4]. In [2] the authors proved that the phenomenon does not occur for the functional

$$
\int_{\Omega} l(|\nabla u(x)|) d x
$$

where $l$ is a convex function, without additional growth or regularity assumptions, provided that both $\partial \Omega$ and $u^{0}$ are smooth. This paper is, to some extent, a sequel to [2]. We consider the more general functional

$$
\int_{\Omega}[l(|\nabla u(x)|)+G(u)] d x
$$

subject to the conditions $u-u^{0} \in W_{0}^{1,1}(\Omega)$, and, under some conditions on the convex functions $l$ and $G$, we prove the nonoccurrence of the Lavrentiev phenomenon, again assuming the smoothness both of $\partial \Omega$ and of $u^{0}$.

2. Notation and a boundedness result. In what follows, by $|A|$ we mean the Lebesgue $N$-dimensional measure of $A \subset \Re^{N}$, and $H^{N-1}(A)$ is the $(N-1)$-dimensional Hausdorff measure of $A$. A vector $x \in \Re^{N}$ will often be written as $\left(\hat{x}, x_{N}\right)$. $\operatorname{Dom}(L)$ is the effective domain of the (possibly extended valued) convex function $L ; L^{*}$ is the polar or Legendre-Fenchel transform of $L . \Omega \subset \Re^{N}$ is a bounded open set, and $\Omega^{\delta}=\{x \in \Omega: d(x, \partial \Omega)<\delta\}$.

\footnotetext{
${ }^{*}$ Received by the editors February 15, 2012; accepted for publication (in revised form) February 4, 2013; published electronically April 18, 2013.

http://www.siam.org/journals/sicon/51-2/86618.html

$\dagger$ Dipartimento di Matematica e Applicazioni, Università di Milano Bicocca, 20125 Milano, Italy (g.bonfanti3@campus.unimib.it, arrigo.cellina@unimib.it).
} 
The following boundedness result will be essential in the proof of the nonoccurrence of the Lavrentiev phenomenon.

Theorem 1. Let $L: \Re^{N} \rightarrow \Re \cup\{+\infty\}$ be an extended-valued, convex, lower semicontinuous function such that Dom $\left(L^{*}\right)=\Re^{N}$. Let $G(u)=\alpha u+g(u)$, with $g: \Re \rightarrow \Re \cup\{+\infty\}$ an extended-valued, convex, lower semicontinuous function with minimum 0 at $u=0$. In addition, assume that at least one of $L$ and $g$ is strictly convex. Let $\Omega$ be bounded. Then, given $U$, there exists $V$ such that for every $\Omega^{\prime} \subset \Omega$, for every $u^{0}$ in $L^{\infty}\left(\Omega^{\prime}\right) \cap W^{1,1}\left(\Omega^{\prime}\right)$ with $\left|u^{0}\right| \leq U$, the solution $\tilde{v}$ to the problem of minimizing

$$
\int_{\Omega^{\prime}}[L(\nabla u(x))+G(u)] d x
$$

on $u-u^{0} \in W_{0}^{1,1}\left(\Omega^{\prime}\right)$ satisfies $|\tilde{v}(x)| \leq V$ a.e. (almost everywhere) in $\Omega^{\prime}$.

Proof. Consider first the case $\alpha=0$. Set $E^{+}=\left\{x \in \Omega^{\prime}:(\tilde{v}-U)^{+}(x)>0\right\}$ and assume that $\left|E^{+}\right|>0$. Since both $U$ and $U+(\tilde{v}-U)^{+}$are in $U+W_{0}^{1,1}\left(\Omega^{\prime}\right)$, the convexity of $L$ implies that

$$
\int_{\Omega^{\prime}} L(\nabla U)=\int_{\Omega^{\prime}} L(0) \leq \int_{\Omega^{\prime}} L\left(\nabla(\tilde{v}-U)^{+}\right),
$$

and hence that $\int_{E^{+}} L(0) \leq \int_{E^{+}} L(\nabla \tilde{v})$ so that, from the properties of $g$, we have $\int_{E^{+}} L(0)+g(U) \leq \int_{E^{+}} L(\nabla \tilde{v})+g(\tilde{v})$. Then, the function $\tilde{v}^{-}=\min \{U, \tilde{v}\}$ is also a solution to the minimization of $(1)$ on $u^{0}+W_{0}^{1,1}\left(\Omega^{\prime}\right)$ and, by convexity, so is $\frac{1}{2} \tilde{v}+\frac{1}{2} \tilde{v}^{-}$, and we obtain that

$$
\text { (2) } \int_{\Omega^{\prime}} L\left(\frac{1}{2} \nabla \tilde{v}+\frac{1}{2} \nabla \tilde{v}^{-}\right)+g\left(\frac{1}{2} \tilde{v}+\frac{1}{2} \tilde{v}^{-}\right)=\frac{1}{2} \int_{\Omega^{\prime}} L(\nabla \tilde{v})+L\left(\nabla \tilde{v}^{-}\right)+\frac{1}{2} \int_{\Omega^{\prime}} g(\tilde{v})+g\left(\tilde{v}^{-}\right)
$$

that gives both $\int_{E^{+}} L\left(\frac{1}{2} \nabla \tilde{v}+\frac{1}{2} \nabla \tilde{v}^{-}\right)=\frac{1}{2} \int_{E^{+}} L(\nabla \tilde{v})+L\left(\nabla \tilde{v}^{-}\right)$and $\int_{E^{+}} g\left(\frac{1}{2} \tilde{v}+\frac{1}{2} \tilde{v}^{-}\right)=$ $\frac{1}{2} \int_{E^{+}} g(\tilde{v})+g\left(\tilde{v}^{-}\right)$. When $g$ is strictly convex, we infer that $\tilde{v}=\tilde{v}^{-}$a.e. on $E^{+}$, and hence that $\left|E^{+}\right|=0$. When $L$ is strictly convex, we obtain that $\nabla \tilde{v}=\nabla \tilde{v}^{-}$a.e. on $E$; apply the Poincaré inequality to the function $\tilde{v}-\tilde{v}^{-}$on $\Omega^{\prime}$ to obtain again that $\tilde{v}=\tilde{v}^{-}$a.e. on $E^{+}$.

Analogously, set $E^{-}=\left\{x \in \Omega^{\prime}:(\tilde{v}+U)^{-}(x)>0\right\}$ to obtain that $\left|E^{-}\right|=0$.

Consider the case $\alpha \neq 0$, and, for definiteness, assume $\alpha>0$.

Again, we have $\tilde{v}(x) \leq U$. Consider the convex function $\frac{N}{\alpha} L^{*}\left(\frac{\alpha}{N} x\right)$, whose domain, by assumption, is $\Re^{N}$ : it is bounded on bounded sets; hence, there exist two constants $c$ and $\tilde{V}$ such that, for $x \in \Omega$,

$$
\tilde{V}+U \leq \frac{N}{\alpha} L^{*}\left(\frac{\alpha}{N} x\right)+U-c \leq 0 .
$$

Call $\tilde{u}$ the restriction of the function $\frac{N}{\alpha} L^{*}\left(\frac{\alpha}{N} x\right)-c$ to $\overline{\left(\Omega^{\prime}\right)}$ so that we have

$$
\tilde{V} \leq \tilde{u}(x) \leq-U
$$

As shown in [3], the function $\tilde{u}$ is a solution to the problem

$$
\operatorname{minimize} \int_{\Omega^{\prime}}[L(\nabla w(x))+\alpha w(x)] d x \text { on } w-\tilde{u} \in W_{0}^{1,1}\left(\Omega^{\prime}\right) .
$$


Consider the functions

$$
w^{+}(x)=\max \{\tilde{v}(x), \tilde{u}(x)\} \text { and } w^{-}(x)=\min \{\tilde{v}(x), \tilde{u}(x)\} ;
$$

since, at $\partial\left(\Omega^{\prime}\right)$, we have $\tilde{u} \leq-U$, then $w^{+} \in u^{0}+W_{0}^{1,1}\left(\Omega^{\prime}\right)$ and $w^{-} \in \tilde{u}+W_{0}^{1,1}\left(\Omega^{\prime}\right)$. Set

$$
E^{*}=\left\{x \in \Omega^{\prime}: \tilde{v}(x)<\tilde{u}(x)\right\}
$$

we have that, a.e. in $\Omega^{\prime}$,

$$
\nabla w^{+}(x)=\left\{\begin{array}{l}
\nabla \tilde{u}(x) \text { on } E^{*}, \\
\nabla \tilde{v}(x) \text { on } \Omega^{\prime} \backslash E^{*},
\end{array} \quad \nabla w^{-}(x)=\left\{\begin{array}{l}
\nabla \tilde{v}(x) \text { on } E^{*} \\
\nabla \tilde{u}(x) \text { on } \Omega^{\prime} \backslash E^{*} .
\end{array}\right.\right.
$$

Since $\tilde{u}$ and $w^{-}$satisfy the same boundary condition and $\tilde{u}$ is a solution to (5), we have

$$
\int_{\Omega^{\prime}}\left[L\left(\nabla w^{-}(x)\right)+\alpha w^{-}(x)\right] d x \geq \int_{\Omega^{\prime}}[L(\nabla \tilde{u}(x))+\alpha \tilde{u}(x)] d x
$$

so that

$$
\int_{E^{*}}[L(\nabla \tilde{v}(x))+\alpha \tilde{v}(x)] d x \geq \int_{E^{*}}[L(\nabla \tilde{u}(x))+\alpha \tilde{u}(x)] d x .
$$

On $E^{*}, \tilde{v}<\tilde{u}<0$, and hence, by the assumptions on $g, g(\tilde{v}(x)) \geq g(\tilde{u}(x))$, and we obtain

$$
\begin{gathered}
\int_{E^{*}}[L(\nabla \tilde{v}(x))+\alpha \tilde{v}(x)+g(\tilde{v}(x))] d x \geq \int_{E^{-}}[L(\nabla \tilde{v}(x))+\alpha \tilde{v}(x)+g(\tilde{u}(x))] d x \\
\geq \int_{E^{*}}[L(\nabla \tilde{u}(x))+\alpha \tilde{u}(x)+g(\tilde{u}(x))] d x
\end{gathered}
$$

The map $w^{+}$is in $u^{0}+W_{0}^{1,1}\left(\Omega^{\prime}\right)$; since

$$
\begin{gathered}
\int_{\Omega^{\prime}}\left[L\left(\nabla w^{+}(x)\right)+\alpha w^{+}(x)+g\left(w^{+}(x)\right)\right] d x \\
=\int_{\Omega^{\prime} \backslash E^{*}}[L(\nabla \tilde{v}(x))+\alpha \tilde{v}(x)+g(\nabla \tilde{v}(x))] d x+\int_{E^{*}}[L(\nabla \tilde{u}(x))+\alpha \tilde{u}(x)+g(\nabla \tilde{u}(x))] d x \\
\leq \int_{\Omega^{\prime} \backslash E^{*}}[L(\nabla \tilde{v}(x))+\alpha \tilde{v}(x)+g(\nabla \tilde{v}(x))] d x+\int_{E^{*}}[L(\nabla \tilde{v}(x))+\alpha \tilde{v}(x)+g(\nabla \tilde{v}(x))] d x,
\end{gathered}
$$

$w^{+}$is a solution to problem (1) on $u^{0}+W_{0}^{1,1}\left(\Omega^{\prime}\right)$. By convexity, so is $\frac{1}{2} \tilde{v}+\frac{1}{2} w^{+}$, and we obtain again

$\int_{\Omega^{\prime}} L\left(\frac{1}{2} \nabla \tilde{v}+\frac{1}{2} \nabla w^{+}\right)+g\left(\frac{1}{2} \tilde{v}+\frac{1}{2} w^{+}\right)=\frac{1}{2} \int_{\Omega^{\prime}} L(\nabla \tilde{v})+L\left(\nabla w^{+}\right)+\frac{1}{2} \int_{\Omega^{\prime}} g(\tilde{v})+g\left(w^{+}\right)$.

As before, the strict convexity assumption yields a contradiction, unless $\left|E^{*}\right|=0$. Hence, a.e. we have $\tilde{v}(x) \geq \tilde{u}(x)$, and, from $\tilde{v}(x) \leq U$, we obtain the boundedness of $\tilde{v}$.

Notice that the minimal area Lagrangian $L(\xi)=\sqrt{1+|\xi|^{2}}$ does not satisfy the assumption that $\operatorname{Dom}\left(L^{*}\right)=\Re^{N}$. For this $L$, problem (1), with $g=0$ and $\alpha$ sufficiently large, admits no solution, and a minimizing sequence is unbounded below. 
3. Assumptions for the nonoccurrence of the Lavrentiev phenomenon and the statement of the main result. In this and the following section we consider convex functions $L$ of the form

$$
L(\xi)=l(|\xi|)
$$

and we call $\tilde{v}$ the solution to the problem of minimizing

$$
\int_{\Omega}[l(|\nabla u(x)|)+G(u)] d x
$$

subject to the condition $u-u^{0} \in W_{0}^{1,1}(\Omega)$. We shall express a regularity condition on $l$ through the function $P$ appearing in the following definition.

Definition 1. Set $P(t)=t l^{\prime}(t)-l(t)$.

$P$ is the polar of the convex function $l$ computed at the value $l^{\prime}$. In the case $l(t)=\frac{1}{p} t^{p}$, we have that $P(t)=\frac{p}{q} l(t)$.

Our next assumption connects the local Lipschitzeanity of $l$ to that of $P$.

Assumption A. $l: \Re \rightarrow \Re$ is a strictly convex, nonnegative, differentiable, symmetric function and such that $\operatorname{Dom}\left(l^{*}\right)=\Re$. Moreover, there exists $K$ such that

$$
|l(t)-l(z)| \leq K|P(t)-P(z)| .
$$

In the case where $l$ is twice differentiable, the above condition takes a simpler form. By the generalized mean value theorem, given $t$ and $z$, there exists $\xi$ such that

$$
\frac{l(t)-l(z)}{P(t)-P(z)}=\frac{l^{\prime}(\xi)}{P^{\prime}(\xi)}=\frac{l^{\prime}(\xi)}{\xi l^{\prime \prime}(\xi)}
$$

and the latter expression is bounded (and condition (6) is verified), for instance, whenever $l$ is a polynomial of degree larger than one or has exponential growth. Again, condition (6) is not verified by the minimal area Lagrangian $l$.

In order to state precisely the regularity assumptions on $\Omega$, we begin by noticing that, in $\Re^{N}$, for every matrix $\left(a_{i, k}\right)$ such that $\left|a_{i, k}\right| \leq \varepsilon$, we have that $\operatorname{det}\left[I+\left(a_{i, k}\right)\right] \rightarrow$ 1 as $\varepsilon \rightarrow 0$. Also, we have that the determinant of any matrix $\left(b_{i, k}+a_{i, k}\right)$, where the $b_{i, k}$ are all 0 with the exception of two 1's on the same line, tends to 0 as $\varepsilon \rightarrow 0$. Hence, the following definition makes sense.

Definition 2. We set $\varepsilon_{0}$ to be such that $\left|a_{i, k}\right| \leq \varepsilon_{0}$ implies that $\operatorname{det}\left[I+\left(a_{i, k}\right)\right] \geq$ $\left(1-\left(\frac{1}{2}\right)^{N}\right)$ and, for $b_{i, k}$ as above, $\operatorname{det}\left[b_{i, k}+a_{i, k}\right] \leq \frac{1}{(N-1) 2^{N}}$.

In what follows, we require that $\partial \Omega$ be of class $C^{2}$. We make precise our request through the following assumption.

Assumption B. $\Omega$ is a bounded open set. There exist $J^{P}$ points $P^{j} \in \partial \Omega$; coordinate systems with the origin in $P^{j}$ and the $\xi_{N}$ axis in the direction of the normal to the inside of $\Omega$; open subsets $V^{j}$ of the tangent plane at $P^{j}$, containing $P^{j}$, and $C^{2}$ functions $\phi^{j}$, with $\phi^{j}(0)=0$ and $\nabla \phi^{j}(0)=0$ such that, as $\hat{\xi}$ ranges over $V^{j}$, $\xi_{N}=\phi^{j}(\hat{\xi})$ describes the surface $\partial \Omega^{j}$, and $\partial \Omega=\cup_{j} \partial \Omega^{j}$. Moreover, all the second derivatives of the functions $\phi^{j}$ are uniformly bounded. For $\hat{\xi} \in V^{j}$, set

$$
\bar{\tau}_{1}=\left(\begin{array}{l}
1 \\
0 \\
\cdot \\
\cdot \\
\phi_{x_{1}}^{j}
\end{array}\right) ; \quad \ldots ; \bar{\tau}_{N-1}=\left(\begin{array}{l}
0 \\
\cdot \\
\cdot \\
\cdot \\
\phi_{x_{N-1}}^{j}
\end{array}\right) ; \quad \bar{\nu}=\left(\begin{array}{l}
-\phi_{x_{1}}^{j} \\
\cdot \\
\cdot \\
\cdot \\
-\phi_{x_{N-1}}^{j} \\
1
\end{array}\right)
$$


and

$$
\nu=\frac{\bar{\nu}}{|\bar{\nu}|} ; \tau_{i}=\frac{\bar{\tau}_{i}}{\left|\bar{\tau}_{i}\right|} .
$$

We assume, for every $j$, that $\left|\bar{\tau}_{i}\right| \leq K^{0}$ and that the matrix $\left(\left\langle\tau_{i}, \tau_{k}\right\rangle\right)$ has determinant $\geq \frac{1}{2}$. Noticing that, for $l<N$, as $\left(\hat{\xi}, \phi^{j}(\hat{\xi})\right) \rightarrow P^{j}$, we have $\left\langle e_{l}, \nu(\hat{\xi})\right\rangle \rightarrow 0$, we shall also assume that $\left|\left\langle e_{l}, \nu(\hat{\xi})\right\rangle\right| \leq \frac{1}{4} \sqrt{\varepsilon_{0}}$ for $\hat{\xi} \in V^{j}$ and $l<N$.

Assumption B in particular implies that $H^{N-1}(\partial \Omega)$ is finite and hence that $|\partial \Omega|=$ 0 . Recall that $\Omega^{\delta}=\{x \in \Omega: d(x, \partial \Omega)<\delta\}$; then, for $\delta>0$ sufficiently small, also $\partial \Omega^{\delta}$ is of class $C^{2}$ and $\left|\partial \Omega^{\delta}\right|=0$.

On $u^{0}$ we shall assume the following.

Assumption C. $u^{0}$ is a $C^{2}$ function on some open set containing $\bar{\Omega}$.

Denote the Hessian matrix of $u^{0}$ by $H_{u^{0}}$. In particular, we shall assume that $u^{0}$ is Lipschitzean of constant $K^{0}$; without loss of generality, we shall assume that $\frac{h^{0}+K^{0}}{h^{0}-K^{0}} \leq 2$.

The following definition is basic to the discussion that follows.

DeFinition 3. For $x \in \Omega$, set

$$
w_{+}^{h}(x)=\min \left\{u^{0}(z)+h|z-x|: z \in \partial \Omega\right\} .
$$

Analogously, set

$$
w_{-}^{h}(x)=\max \left\{u^{0}(z)-h|z-x|: z \in \partial \Omega\right\} .
$$

As a consequence of Assumption B we obtain, in particular, that there exists $\delta$ and $h^{0}$ such that, for all $h \geq h^{0}$, for any $x \in \Omega$ with $d(x, \partial \Omega) \leq \delta$, there exists a unique $y_{h}(x)$ in $\partial \Omega$ such that

$$
\min \left\{u^{0}(z)+h|z-x|: z \in \partial \Omega\right\}=u^{0}\left(y_{h}(x)\right)+h\left|y_{h}(x)-x\right| .
$$

Then, for each $j$, for every $h \geq h^{0}$, set $\left(\Omega_{h}^{+}\right)_{j}=\left\{x \in \Omega: y_{h}(x) \in \partial \Omega_{j}\right.$ and $\tilde{v}(x)>$ $\left.w_{+}^{h}(x)\right\}$. For $x \in\left(\Omega_{h}^{+}\right)_{j}$, there exists a unique $\hat{\xi}(x) \in V^{j}$ such that $y_{h}(x)=\left(\hat{\xi}, \phi_{j}(\hat{\xi})\right)$.

It will be our purpose to prove the following theorem on the nonoccurrence of Lavrentiev's phenomenon.

Theorem 2. Let Assumptions A, B, and $\mathrm{C}$ hold. Let $G$ be as in Theorem 1. Let $\tilde{v}$ be a solution to the problem of minimizing the functional

$$
\int_{\Omega}[l(|\nabla u(x)|)+G(u(x))] d x
$$

on $u-u^{0} \in W_{0}^{1,1}(\Omega)$. Then, given $\varepsilon$, there exists a Lipschitzean function $v^{L}$ such that $v^{L}-\tilde{v} \in W_{0}^{1,1}(\Omega)$ and

$$
\int_{\Omega}\left[l\left(\left|\nabla v^{L}(x)\right|\right)+G\left(v^{L}(x)\right)\right] d x-\int_{\Omega}[l(|\nabla \tilde{v}(x)|)+G(\tilde{v}(x))] d x \leq \varepsilon .
$$

4. Differentiability properties. (a) As shown in [2], $u^{0}, w_{+}^{h}$, and the map $x \rightarrow \hat{\xi}$ (that depends on $j$ and $h$ ) are connected through the identities that follow.

For every $j$, for $i=1, \ldots, N-1$, we have that

$$
\left\langle\nabla w_{+}^{h}\left(\hat{\xi}, \phi^{j}(\hat{\xi})\right), \tau_{i}\right\rangle=\left\langle\nabla u^{0}\left(\hat{\xi}, \phi^{j}(\hat{\xi})\right), \tau_{i}\right\rangle .
$$


Let $a_{i}=a_{i}(\hat{\xi}), i=1, \ldots, N-1$, be the solution to the linear system

$$
\left\langle\nabla u^{0}\left(\hat{\xi}, \phi^{j}(\hat{\xi})\right), \tau_{k}(\hat{\xi})\right\rangle=\sum_{i} a_{i}(\hat{\xi})\left\langle\tau_{i}(\hat{\xi}), \tau_{k}(\hat{\xi})\right\rangle ;
$$

a solution exists, and it is unique by Assumption B; moreover, since the determinant of $\left\langle\tau_{i}(\hat{\xi}), \tau_{k}(\hat{\xi})\right\rangle$ is bounded away from zero, uniformly with respect to $\hat{\xi}$ and $j$, all the $\left|a_{i}(\hat{\xi})\right|$ are bounded by some real $\alpha$. From (10) and the fact that the norm of $\nabla w_{+}^{h}$ is $h$, we obtain (see [2])

$$
\left\langle\nabla w_{+}^{h}, \nu\right\rangle=\sqrt{h^{2}-\left(\sum_{i, l=1, \ldots, N-1} a_{i} a_{l}\left\langle\tau_{i}, \tau_{l}\right\rangle\right)} .
$$

Let $b_{i}^{l}$ be the solution to the linear system

$$
\left\langle e_{l}, \tau_{k}(\hat{\xi})\right\rangle=\sum_{i} b_{i}^{l}(\hat{\xi})\left\langle\tau_{i}(\hat{\xi}), \tau_{k}(\hat{\xi})\right\rangle
$$

again solutions are unique and uniformly bounded by some $\beta$. We obtain, from [2], the identity

$$
\equiv\left[\left\langle e_{l}, \nu\right\rangle \sqrt{\left.h^{2}-\left(\sum_{i, k=1, \ldots, N-1} a_{i} a_{k}, e_{l}\right\rangle\left(\tau_{i}, \tau_{k}\right\rangle\right)}+\sum_{i=1}^{N-1} b_{i}^{l}\left\langle\nabla u^{0}, \tau_{i}\right\rangle\right],
$$

where on the right-hand side the $a_{i}, b_{i}, \tau_{i}$ are computed at $\hat{\xi}$, and $\nabla u^{0}$ is computed at $\left(\hat{\xi}, \phi^{j}(\hat{\xi})\right)$. In particular, from the boundedness of $a_{i}$ and $b_{i}$, we have that there exists $h^{1} \geq h^{0}$ such that, for $h \geq h^{1}$,

$$
\frac{1}{h}\left|\left\langle\nabla w_{+}^{h}, e_{l}\right\rangle\right| \leq 2\left|\left\langle e_{l}, \nu\right\rangle\right|+\frac{\beta K^{0}}{h} .
$$

For brevity, set $\left(w_{+}^{h}\right)_{l}^{\prime}=\left\langle\nabla w_{+}^{h}, e_{l}\right\rangle$; the second identity we have is

$$
\left(w_{+}^{h}\right)_{j}^{\prime}(x) \equiv\left(w_{+}^{h}\right)_{j}^{\prime}(\hat{\xi}(x), \phi(\hat{\xi}(x)))
$$

and the third identity is

$$
\left(\begin{array}{l}
\hat{x} \\
x_{N}
\end{array}\right) \equiv\left(\begin{array}{l}
\hat{\xi}(x) \\
\phi(\hat{\xi}(x))
\end{array}\right)+\frac{\left(w_{+}^{h}(x)-u^{0}(\hat{\xi}(x), \phi(\hat{\xi}(x)))\right)}{h} \frac{\nabla w_{+}^{h}(\hat{\xi}(x), \phi(\hat{\xi}(x))}{h}
$$

(b) Differentiating both sides of (11), we obtain

$$
\left(\bar{\tau}_{j}\right)^{T} H_{u^{0}} \bar{\tau}_{l}+u_{\xi_{N}}^{0} \phi_{\xi_{j} \xi_{l}}-\sum_{i} a_{i} \frac{\partial}{\partial \xi_{l}}\left\langle\tau_{i}, \bar{\tau}_{j}\right\rangle=\sum_{i}\left(a_{i}\right)_{\xi_{l}}\left\langle\tau_{i}, \bar{\tau}_{j}\right\rangle ;
$$

the left-hand side is bounded, while the determinant of the coefficients is uniformly bounded away from zero, and hence all the $\left(a_{i}\right)_{\xi_{l}}$ are uniformly bounded. 
From (13) we have $\left\langle e_{l}, \bar{\tau}_{k}(\hat{\xi})\right\rangle=\sum_{i} b_{i}^{l}(\hat{\xi})\left\langle\tau_{i}(\hat{\xi}), \bar{\tau}_{k}(\hat{\xi})\right\rangle$ and, differentiating,

$$
\left\langle e_{j}, \frac{\partial}{\partial \xi_{l}} \bar{\tau}_{k}\right\rangle-\sum_{i} b_{i}^{l} \frac{\partial}{\partial \xi_{l}}\left\langle\tau_{i}, \bar{\tau}_{k}\right\rangle=\sum_{i}\left(b_{i}^{l}\right)_{\xi_{l}}\left\langle\tau_{i}, \bar{\tau}_{k}\right\rangle
$$

again, all the $\left(b_{i}^{l}\right)_{\xi_{l}}$ are uniformly bounded.

(c) We can differentiate identity (14) to obtain

$$
\begin{gathered}
\frac{\partial}{\partial \xi_{l}}\left\langle\nabla w_{+}^{h}, e_{j}\right\rangle=\left(\left\langle e_{j}, \nu\right\rangle\right)_{\xi_{l}} \sqrt{h^{2}-\left(\sum a_{i} a_{j}\left\langle\tau_{i}, \tau_{j}\right\rangle\right)} \\
-\left\langle e_{j}, \nu\right\rangle \frac{\frac{\partial}{\partial \xi_{l}}\left(\sum a_{i} a_{j}\left\langle\tau_{i}, \tau_{j}\right\rangle\right)}{2 \sqrt{h^{2}-\left(\sum a_{i} a_{j}\left\langle\tau_{i}, \tau_{j}\right\rangle\right)}}+\sum_{i=1, \ldots, N-1}\left(b_{i}^{j}\left\langle\nabla u^{0}, \tau_{i}\right\rangle\right)_{\xi_{l}} .
\end{gathered}
$$

We have that $\left.\mid\left\langle e_{j}, \nu\right\rangle\right)_{\xi_{l}} \mid$ is bounded; the denominator of the second term is bounded away from zero for all $h$ sufficiently large; the third term is bounded. Hence, there exists $h^{2} \geq h^{1}, \alpha^{1}$, and $\beta^{1}$ such that the absolute value of the right-hand side is bounded by $\alpha^{1} h+\beta^{1}$ for all $h \geq h^{2}$.

(d) Differentiating the first $N-1$ lines of (17) with respect to $x_{j}$, we obtain

$$
\begin{array}{r}
\delta_{i, j}=\xi_{x_{j}}^{i}+\frac{1}{h^{2}}\left[\left(\left(w_{+}^{h}\right)_{x_{j}}-\sum_{l}\left\langle\nabla u^{0}, \bar{\tau}_{l}\right\rangle\left(\xi^{l}\right)_{x_{j}}\right)\left(w_{+}^{h}\right)_{x_{i}}\right. \\
\left.+\left(w_{+}^{h}-u^{0}\right) \sum_{l}\left\langle\nabla\left(\left(w_{+}^{h}\right)_{i}^{\prime}\right), \bar{\tau}_{l}\right\rangle \xi_{x_{j}}^{l}\right],
\end{array}
$$

where $\left\langle\nabla\left(\left(w_{+}^{h}\right)_{i}^{\prime}\right), \bar{\tau}_{l}\right\rangle, u^{0}$, and $\left\langle\nabla u^{0}, \bar{\tau}_{l}\right\rangle$ are computed at the point $(\hat{\xi}, \phi(\hat{\xi}))$.

System (21) has the form

$$
\begin{gathered}
\left(\begin{array}{llll}
1+\sigma_{1,1} & \cdot & \sigma_{1, N-1} & \sigma_{1, N} \\
\cdot & \cdot & \cdot & \cdot \\
\sigma_{N-1,1} & \cdot & 1+\sigma_{N-1, N-1} & \sigma_{N-1, N}
\end{array}\right) \\
=\left(\begin{array}{llll}
\left(1+\eta_{1,1}\right) & \cdot & \eta_{1, N-1} \\
\cdot & \cdot & \cdot \\
\eta_{N-1,1} & \cdot & \left(1+\eta_{N-1, N-1}\right)
\end{array}\right)\left(\begin{array}{llll}
\xi_{x_{1}}^{1} & \xi_{x_{2}}^{1} & \cdot & \xi_{x_{N}}^{1} \\
\dot{\xi}_{x_{1}}^{N-1} & \xi_{x_{2}}^{N-1} & \cdot & \xi_{x_{N}}^{N-1}
\end{array}\right)
\end{gathered}
$$

with

$$
\eta_{i, l}=\frac{1}{h^{2}}\left[\left(w_{+}^{h}-u^{0}\right)\left\langle\nabla\left(\left(w_{+}^{h}\right)_{i}^{\prime}\right), \bar{\tau}_{l}\right\rangle-\left(w_{+}^{h}\right)_{x_{i}}\left\langle\nabla u^{0}, \bar{\tau}_{l}\right\rangle\right]
$$

and $\sigma_{i, l}=-\frac{1}{h^{2}}\left(w_{+}^{h}\right)_{x_{i}}\left(w_{+}^{h}\right)_{x_{l}}$, where, recalling (16), the $\left(w_{+}^{h}\right)_{x_{i}}$ are computed at $(\hat{\xi}(x), \phi(\hat{\xi}(x)))$.

We wish to estimate the terms $\eta_{i, l}$ in order to show that system (22) is solvable in the unknowns $\xi_{x_{i}}^{k}$. We have $\left|\left(w_{+}^{h}\right)_{x_{i}}\left\langle\nabla u^{0}, \bar{\tau}_{l}\right\rangle\right| \leq h K^{0}$ and $\left\langle\nabla\left(\left(w_{+}^{h}\right)_{i}^{\prime}\right), \bar{\tau}_{l}\right\rangle \leq K^{0}\left(\alpha^{1} h+\right.$ $\left.\beta^{1}\right)$. The term $w_{+}^{h}(x)-u^{0}(y(x))$ will be computed only on the set of points where 
$\left|w_{+}^{h}(x)-u^{0}(y(x))\right| \leq U+V$; hence, there exists $h^{3} \geq h^{2}$ such that, for $h \geq h^{3}$, the determinant of the matrix of coefficients on the right-hand side of (22) is between $\frac{4}{5}$ and $\frac{6}{5}$ and such that $\left|\eta_{i, l}\right| \leq \varepsilon_{0}$. Hence, the solutions $\xi_{x_{i}}^{k}$ exist and are bounded; in particular, the Jacobian $J$ of the transformation $x \rightarrow \hat{\xi}$ is bounded. We wish to show that it is bounded away from 0 . Consider the matrix $\left(\xi_{x_{i}}^{k}\right), 1 \leq k \leq N-1,1 \leq i \leq N$. By the formula for the Jacobian [5, page 89], it is enough to show that the absolute value of one of the determinants of the $(N-1) \times(N-1)$ matrices extracted from $\left(\xi_{x_{i}}^{k}\right)$ is bounded away from 0 .

Consider the matrix $\left(\xi_{x_{i}}^{k}\right), 1 \leq k \leq N-1,1 \leq i \leq N-1$. Let $h^{4} \geq h^{3}$ be such that $\frac{\beta K^{0}}{h} \leq \frac{1}{2} \sqrt{\varepsilon_{0}}$ for every $h \geq h^{4}$ so that, by Assumption B and (15), we have $\left|\sigma_{i, k}\right| \leq \varepsilon_{0}$. Recalling Definition 2 we obtain that, for $k=1, \ldots, N-1$, $\left(\xi_{x_{k}}^{k}\right) \geq \frac{5}{6}\left(1-\left(\frac{1}{2}\right)^{N}\right)$. Consider $\xi_{x_{i}}^{k}$ for $i \neq k$. Substituting the $k$ th column on the left-hand side of (22) in the $i$ th column of the coefficient matrix, we can apply the second statement in Definition 2 to obtain that $\left|\xi_{x_{i}}^{k}\right| \leq \frac{5}{4} \frac{1}{(N-1) 2^{N}}$. Hence, the matrix $\left(\xi_{x_{i}}^{k}\right)$ has terms at least $\frac{5}{6}\left(1-\left(\frac{1}{2}\right)^{N}\right)$ on the diagonal and terms in absolute value not larger than $\frac{5}{4} \frac{1}{(N-1) 2^{N}}$ off the diagonal. The determinant of this matrix is at least $\left(\frac{5}{6}\left(1-\left(\frac{1}{2}\right)^{N}\right)\right)^{N}-(N-1)\left(\frac{5}{4}\right)^{N}\left(\frac{1}{(N-1) 2^{N}}\left(1-\left(\frac{1}{2}\right)^{N}\right)^{N-1}\right)$, that is strictly positive for every $N \geq 2$. Hence, we have proved that there exist $m, M>0$ such that, for every $h \geq h^{4}$,

$$
m \leq J \leq M
$$

5. Approximating with the function $M^{h}$. We shall need the following definition.

Definition 4. Set

$$
M^{h}(x)= \begin{cases}w_{+}^{h}(x) & \text { when } \tilde{v}(x)>w_{+}^{h}(x) \\ \tilde{v}(x) & \text { when } w_{-}^{h}(x) \leq \tilde{v}(x) \leq w_{+}^{h}(x), \\ w_{-}^{h}(x) & \text { when } \tilde{v}(x)<w_{-}^{h}(x) .\end{cases}
$$

Theorem 3. Let Assumptions A, B, and $\mathrm{C}$ hold. Let $G$ be as in Theorem 1 and $\tilde{v}$ as in Theorem 2. Then, given $\sigma$, there exists $\tilde{h}$ such that $h \geq \tilde{h}$ implies

$$
\int_{\Omega}\left[l\left(\left|\nabla M^{h}(x)\right|\right)+G\left(M^{h}(x)\right)\right] d x-\int_{\Omega}[l(|\nabla \tilde{v}(x)|)+G(\tilde{v}(x))] d x \leq \sigma .
$$

Proof. Consider first the function $w_{+}^{h}$ for $h \geq h^{4}$. Fix $j$ and consider $\left(\Omega_{h}^{+}\right)_{j}$ with the coordinate system centered at $P_{j}$, as described in Assumption B. Apply the coarea theorem to the map $x \rightarrow \hat{\xi}$, whose Jacobian will be denoted by $J$, to obtain

$$
\int_{\left(\Omega_{h}^{+}\right)_{j}}[l(|\nabla \tilde{v}(x)|)+G(\tilde{v}(x))] d x=\int_{\Re^{N-1}}\left[\int_{\{\hat{\xi}=\hat{z}\} \cap\left(\Omega_{h}^{+}\right)_{j}} \frac{[l(|\nabla \tilde{v}(x)|)+G(\tilde{v}(x))]}{J} d H^{1}\right] d \hat{z} .
$$

Set $z=\left(\hat{z}, \phi_{j}(\hat{z})\right)$; parametrize the line segment $\{\hat{\xi}(x)=\hat{z}\}$ as $z+\ell \frac{x-z}{|x-z|}$. For almost every $\hat{z}$, the map $\ell \rightarrow \tilde{v}\left(z+\ell \frac{x-z}{|x-z|}\right)$ is absolutely continuous. For any such $\hat{z}$, there are at most countably many open intervals $\left(a_{i}, b_{i}\right)$ such that, for $\ell \in \cup\left(a_{i}, b_{i}\right)$, we have $w_{+}^{h}\left(z+\ell \frac{x-z}{|x-z|}\right)<\tilde{v}\left(z+\ell \frac{x-z}{|x-z|}\right), w_{+}^{h}\left(z+a_{i} \frac{x-z}{|x-z|}\right)=\tilde{v}\left(z+a_{i} \frac{x-z}{|x-z|}\right)$, and $w_{+}^{h}\left(z+b_{i} \frac{x-z}{|x-z|}\right)=\tilde{v}\left(z+b_{i} \frac{x-z}{x-z \mid}\right)$ so that

$$
\frac{\tilde{v}\left(z+b_{i} \frac{x-z}{x-z \mid}\right)-\tilde{v}\left(z+a_{i} \frac{x-z}{\mid x-z}\right)}{b_{i}-a_{i}}=h .
$$


Moreover, apply Theorem 1 to the solution $\tilde{v}$ and to $\Omega^{\prime}=\Omega$ to infer the existence of $V$ such that $|\tilde{v}| \leq V$ on $\Omega$. In particular, this implies that $\left|\tilde{v}\left(a_{i}\right)\right| \leq V$ and $\left|\tilde{v}\left(b_{i}\right)\right| \leq V$. Since $w_{+}^{h}(z)=u^{0}(z) \geq-U$ and the gradient of $w_{+}^{h}$ has norm $h$ and direction $\frac{x-z}{|x-z|}$, all the points in $\left\{z+\ell \frac{x-z}{|x-z|}\right\} \cap\left(\Omega_{h}^{+}\right)_{j}$, including $\ell=a_{i}$ and the $\ell=b_{i}$, are described by $\ell$ in the interval $\left[0, \frac{U+V}{h}\right]$, i.e., have a distance from $z$ at most $\frac{U+V}{h}$. Since this estimate is independent on $z$ and $j$, it follows that every point in $\Omega_{h}^{+}$has a distance from $\partial \Omega$ at most $\frac{U+V}{h}$ so that, in particular, we have

$$
\Omega_{h}^{+} \subset \Omega^{\frac{U+V}{h}} .
$$

We have

$$
\frac{d}{d \ell} \tilde{v}\left(z+\ell \frac{x-z}{|x-z|}\right)=\left\langle\nabla \tilde{v}\left(z+\ell \frac{x-z}{|x-z|}\right), \frac{x-z}{|x-z|}\right\rangle
$$

so that

$$
\left|\frac{d}{d \ell} \tilde{v}\left(z+\ell \frac{x-z}{|x-z|}\right)\right| \leq\left|\nabla \tilde{v}\left(z+\ell \frac{x-z}{|x-z|}\right)\right|
$$

and, by the assumptions on $l$,

$$
l\left(\left|\frac{d}{d \ell} \tilde{v}\left(z+\ell \frac{x-z}{|x-z|}\right)\right|\right) \leq l\left(\left|\nabla \tilde{v}\left(z+\ell \frac{x-z}{|x-z|}\right)\right|\right) .
$$

Hence, for each $i$,

$$
\begin{aligned}
& \int_{a_{i}}^{b_{i}}\left[l\left(\left|\nabla \tilde{v}\left(z+\ell \frac{x-z}{|x-z|}\right)\right|\right)+G\left(\tilde{v}\left(z+\ell \frac{x-z}{|x-z|}\right)\right)\right] d \ell \\
\geq & \int_{a_{i}}^{b_{i}}\left[l\left(\left|\frac{d}{d \ell} \tilde{v}\left(z+\ell \frac{x-z}{|x-z|}\right)\right|\right)+G\left(\tilde{v}\left(z+\ell \frac{x-z}{|x-z|}\right)\right)\right] d \ell .
\end{aligned}
$$

For fixed $i$, consider the problem of minimizing

$$
\int_{a_{i}}^{b_{i}}\left[l\left(\left|v^{\prime}(\ell)\right|\right)+G(v(\ell))\right] d \ell, \quad v\left(a_{i}\right)=\tilde{v}\left(a_{i}\right), \quad v\left(b_{i}\right)=\tilde{v}\left(b_{i}\right),
$$

and let $\tilde{u}_{i}$ be the solution so that, in particular,

$$
\begin{gathered}
\int_{a_{i}}^{b_{i}}\left[l\left(\left|\tilde{u}_{i}^{\prime}(\ell)\right|\right)+G\left(\tilde{u}_{i}(\ell)\right)\right] d \ell \\
\leq \int_{a_{i}}^{b_{i}}\left[l\left(\left|\frac{d}{d \ell} \tilde{v}\left(z+\ell \frac{x-z}{|x-z|}\right)\right|\right)+G\left(\tilde{v}\left(z+\ell \frac{x-z}{|x-z|}\right)\right)\right] d \ell ;
\end{gathered}
$$

under our assumptions, $\tilde{u}_{i}$ is a differentiable function, and, by (24) and the mean value theorem, there exists $\ell_{i} \in\left(a_{i}, b_{i}\right)$ such that $\tilde{u}_{i}^{\prime}\left(\ell_{i}\right)=h$. The du Bois-Reymond condition yields the existence of a constant $c_{i}$ such that

$$
\left|\tilde{u}_{i}^{\prime}(\ell)\right| l^{\prime}\left(\left|\tilde{u}_{i}^{\prime}(\ell)\right|\right)-l\left(\left|\tilde{u}_{i}^{\prime}(\ell)\right|\right)-G\left(\tilde{u}_{i}(\ell)\right)=c_{i},
$$


and hence, in particular, recalling Definition 1,

$$
c_{i}=h l^{\prime}(h)-\left(l(h)+G\left(\tilde{u}_{i}\left(\ell_{i}\right)\right)\right)=P(h)-G\left(\tilde{u}_{i}\left(\ell_{i}\right)\right)
$$

so that

$$
P\left(\left|\tilde{u}_{i}^{\prime}(\ell)\right|\right)-P(h)=G\left(\tilde{u}_{i}(\ell)\right)-G\left(\tilde{u}_{i}\left(\ell_{i}\right)\right)
$$

Apply Theorem 1 to $\tilde{u}_{i}$ for $N=1, \Omega=(0, \operatorname{diam}(\Omega)), U=V$, and $\left(a_{i}, b_{i}\right)=\Omega^{\prime}$ to infer the existence of $\tilde{V}$ such that we have $\left|\tilde{u}_{i}(\ell)\right| \leq \tilde{V}$; since the function $G$ maps bounded sets to bounded sets, there exists $\Gamma$ such that $\left|G\left(\tilde{u}_{i}(\ell)\right)-G\left(\tilde{u}_{i}\left(\ell_{i}\right)\right)\right| \leq \Gamma$ and $\left|G\left(\tilde{u}_{i}(\ell)\right)\right| \leq \Gamma,\left|G\left(w_{+}^{h}(\ell)\right)\right| \leq \Gamma,|G(\tilde{v})| \leq \Gamma$. Since $V$ does not depend on $h, j$, and $i$, neither does $\Gamma$.

Assumption A yields

$$
\left|l\left(\tilde{u}_{i}^{\prime}(\ell)\right)-l(h)\right| \leq K\left|P\left(\tilde{u}_{i}^{\prime}(\ell)\right)-P(h)\right| \leq K \Gamma ;
$$

recalling (26) and (27), we have

$$
\begin{gathered}
\int_{a_{i}}^{b_{i}}\left[l\left(\left|\nabla \tilde{v}\left(z+\ell \frac{x-z}{|x-z|}\right)\right|\right)+G\left(\tilde{v}\left(z+\ell \frac{x-z}{|x-z|}\right)\right)\right] d \ell \geq \int_{a_{i}}^{b_{i}}\left[l\left(\left|\tilde{u}_{i}^{\prime}(\ell)\right|\right)+G\left(\tilde{u}_{i}(\ell)\right)\right] d \ell \\
=\int_{a_{i}}^{b_{i}}\left[l(h)+l\left(\left|\tilde{u}_{i}^{\prime}(\ell)\right|\right)-l(h)+G\left(\tilde{u}_{i}(\ell)\right)\right] d \ell \geq \int_{a_{i}}^{b_{i}}[l(h)-K \Gamma-\Gamma] d \ell .
\end{gathered}
$$

The last inequality yields

$$
\int_{a_{i}}^{b_{i}} l(h) d \ell \leq \int_{a_{i}}^{b_{i}}\left[l\left(\left|\nabla \tilde{v}\left(z+\ell \frac{x-z}{|x-z|}\right)\right|\right)+(K+2) \Gamma\right] d \ell
$$

so that, recalling (23),

$$
\begin{gathered}
\int_{\left(\Omega_{h}^{+}\right)_{j}} l(h) d x=\int_{\Re^{N-1}}\left[\sum_{i} \int_{a_{i}}^{b_{i}} \frac{l(h)}{J} d \ell\right] d \hat{z} \leq \frac{1}{m} \int_{\Re^{N-1}}\left[\sum_{i} \int_{a_{i}}^{b_{i}} l(h) d \ell\right] d \hat{z} \\
\leq \frac{1}{m} \int_{\Re^{N-1}}\left[\sum_{i} \int_{a_{i}}^{b_{i}}\left[l\left(\left|\nabla \tilde{v}\left(z+\ell \frac{x-z}{|x-z|}\right)\right|\right)+(K+2) \Gamma\right] d \ell\right] d \hat{z} \\
\leq \frac{M}{m} \int_{\Re^{N-1}}\left[\sum_{i} \int_{a_{i}}^{b_{i}} \frac{\left[l\left(\left|\nabla \tilde{v}\left(z+\ell \frac{x-z}{|x-z|}\right)\right|\right)+(K+2) \Gamma\right]}{J} d \ell\right] d \hat{z} \\
=\frac{M}{m} \int_{\left(\Omega_{h}^{+}\right)_{j}}[l(|\nabla \tilde{v}(x)|)+(K+2) \Gamma] d x .
\end{gathered}
$$


From (29) and the boundedness of $G(\tilde{v})$ and of $G\left(w_{+}^{h}\right)$, we obtain the main estimate of this section:

$$
\begin{aligned}
& \int_{\Omega_{h}^{+}}\left[l(h)+G\left(w_{+}^{h}\right)\right] d x \leq \sum_{j=1}^{J^{P}} \int_{\left(\Omega_{h}^{+}\right)_{j}}\left[l(h)+G\left(w_{+}^{h}\right)\right] d x \\
& \leq \sum_{j=1}^{J^{P}} \frac{M}{m} \int_{\left(\Omega_{h}^{+}\right)_{j}}[l(|\nabla \tilde{v}(x)|)+G(\tilde{v})+(K+4) \Gamma] d x \\
& \leq J^{P} \frac{M}{m} \int_{\left(\Omega_{h}^{+}\right)}[l(|\nabla \tilde{v}(x)|)+G(\tilde{v})+(K+4) \Gamma] d x .
\end{aligned}
$$

Since $\int_{\Omega}[l(|\nabla \tilde{v}(x)|)] d x<\infty$, from (25) we infer that

$$
\lim _{h \rightarrow \infty} \int_{\Omega_{h}^{+}}\left[l(h)+G\left(w_{+}^{h}\right)\right] d x=0 .
$$

Consider now the function $w_{-}^{h}$ in a similar way to obtain the result.

6. Smoothing and the definition of $v^{L}$. Let us begin by smoothing the function $M^{h}$. We shall need the following lemmas from [2].

Lemma 1. Let $\Omega$ and $u^{0}$ be as in Theorem 1, let $y_{h}$ be defined as in (9), and let $h \geq h^{0}$. We have $\left|y_{h}(x)-x\right| \leq \frac{h+K^{0}}{h-K^{0}} d(x, \partial \Omega) \leq 2 d(x, \partial \Omega)$.

Lemma 2. Let $v \in W^{1,1}(\Omega)$ be such that $|v(x)| \leq V$ a.e. on $\Omega$, and, on $\Omega \backslash \Omega^{\delta}$, define the function

$$
\tilde{v}(x)=\frac{1}{|B(x, \delta)|} \int_{B(x, \delta)} v(z) d z .
$$

Then (i) $\tilde{v}$ is Lipschitzean of constant $N V \frac{1}{\delta}$, and (ii) $\tilde{v}$ is a.e. differentiable, and, at a point $x$ of differentiability, we have $\nabla \tilde{v}(x)=\frac{1}{|B(x, \delta)|} \int_{B(x, \delta)} \nabla v(z) d z$.

Lemma 3. Assume that either (i) $f$ is measurable and such that $|f(x)| \leq$ $D d(x, \partial \Omega)$, or (ii) $f$ is Lipschitzean with Lipschitz constant D. Then, there exists $D^{*}$ such that the function

$$
\tilde{f}(x)=\frac{1}{|B(x, d(x, \partial \Omega))|} \int_{B(x, d(x, \partial \Omega))} f(z) d z
$$

is Lipschitzean of constant $D^{*}$.

Proof of Theorem 2. Fix $\varepsilon$. In Theorem 3 set $\sigma=\frac{\varepsilon}{2}$ and consider the corresponding $\tilde{h}$. For $h \geq \tilde{h}$, write

$$
M^{h}(x)=u^{0}(x)+\left(M^{h}(x)-M^{h}\left(y_{h}(x)\right)\right)-\left(u^{0}(x)-u^{0}\left(y_{h}(x)\right)\right) ;
$$

we have $\left|M^{h}(x)-M^{h}\left(y_{h}(x)\right)-\left(u^{0}(x)-u^{0}\left(y_{h}(x)\right)\right)\right| \leq(h+K)\left|y_{h}(x)-x\right| \leq 2(h+$ $K) d(x, \partial \Omega)$ by Lemma 1 . Hence, $M^{h}$ is the sum of the Lipschitzean function $u^{0}$ and of a function $f$ such that $|f(x)| \leq D d(x, \partial \Omega)$ : Lemma 3 can be applied to obtain that $\tilde{M}^{h}$ (as defined in Lemma 3) is Lipschitzean of Lipschitz constant $D^{*}$. 
Noticing that $\sup \left|\tilde{M}^{h}\right| \leq \sup \left|M^{h}\right| \leq V$, let $\delta^{*}$ be such that

$$
\int_{\Omega^{\delta^{*}}}\left[l\left(\left|D^{*}\right|\right)+G(V)\right] \mathrm{d} x \leq \frac{\varepsilon}{3},
$$

and define the Lipschitzean function

$$
v^{L}(x)=\left\{\begin{array}{cc}
\tilde{M}^{h}(x) & \text { when } d(x, \partial \Omega) \leq \delta^{*} \\
\frac{1}{\left|B\left(x, \delta^{*}\right)\right|} \int_{B\left(x, \delta^{*}\right)} M^{h}(z) \mathrm{d} z & \text { when } d(x, \partial \Omega)>\delta^{*}
\end{array}\right.
$$

On $\Omega^{\delta^{*}}$, from Lemma 2, we have

$$
\nabla v^{L}(x)=\frac{1}{\left|B\left(0, \delta^{*}\right)\right|} \int_{B\left(0, \delta^{*}\right)} \nabla M^{h}(x-z) \mathrm{d} z
$$

so that

$$
\begin{aligned}
& \int_{\Omega^{\delta^{*}}} l\left(\left|\nabla v^{L}(x)\right|\right) \mathrm{d} x=\int_{\Omega^{\delta^{*}}}\left[l\left(\left|\frac{1}{\left|B\left(0, \delta^{*}\right)\right|} \int_{B\left(0, \delta^{*}\right)} \nabla M^{h}(x-z) \mathrm{d} z\right|\right)\right] \mathrm{d} x \\
& \leq \frac{1}{\left|B\left(0, \delta^{*}\right)\right|} \int_{B\left(0, \delta^{*}\right)}\left(\int_{\Omega^{\delta^{*}}} l\left(\left|\nabla M^{h}(x-z)\right|\right) \mathrm{d} x\right) \mathrm{d} z \leq \int_{\Omega} l\left(\left|\nabla M^{h}(x)\right|\right) \mathrm{d} x .
\end{aligned}
$$

Analogously, we obtain

$$
\int_{\Omega^{\delta^{*}}} G\left(v^{L}(x)\right) \mathrm{d} x \leq \int_{\Omega} G\left(M^{h}(x)\right) \mathrm{d} x
$$

so that

$$
\int_{\Omega}\left[l\left(\left|\nabla v^{L}(x)\right|\right)+G\left(v^{L}(x)\right)\right] \mathrm{d} x \leq \frac{\varepsilon}{2}+\int_{\Omega}\left[l\left(\left|\nabla M^{h}(x)\right|\right)+G\left(M^{h}(x)\right)\right] \mathrm{d} x,
$$

and, by the choice of $\sigma$, we obtain

$$
\int_{\Omega}\left[l\left(\left|\nabla v^{L}(x)\right|\right)+G\left(v^{L}(x)\right)\right] \mathrm{d} x \leq \int_{\Omega}[l(|\nabla \tilde{v}(x)|)+G(\tilde{v}(x))] \mathrm{d} x+\varepsilon .
$$

\section{REFERENCES}

[1] G. Alberti and F. Serra Cassano, Non-occurrence of gap for one-dimensional autonomous functionals, in Calculus of Variations, Homogenization and Continuum Mechanics (Marseille, 1993), Ser. Adv. Math. Appl. Sci. 18, World Sci. Publ., River Edge, NJ, 1994, pp. 1-17.

[2] G Bonfanti and A. Cellina, On the non-occurrence of the Lavrentiev phenomenon, Adv. Calc. Var., 6 (2013), pp. 93-121.

[3] A. Cellina, Uniqueness and comparison results for functionals depending on $\nabla u$ and on $u$, SIAM J. Optim., 18 (2007), pp. 711-716.

[4] L. Esposito, F. Leonetti, And G. Mingione, Regularity for minimizers of functionals with p-q growth, NoDEA Nonlinear Differential Equations Appl., 6 (1999), pp. 133-148.

[5] L. C. Evans and R. F. Gariepy, Measure Theory and Fine Properties of Functions, Stud. Adv. Math., CRC Press, Boca Raton, FL, 1992.

[6] M. Lavrentiev, Sur quelques problèmes du calcul des variations, Ann. Mat. Pura Appl., 4 (1927), pp. 9-28. 